\title{
ZNF367 is differentially expressed in metastasis to lymph nodes in human breast cancer.
}

Shahan Mamoor, $\mathrm{MS}^{1}$

${ }^{1}$ shahanmamoor@gmail.com

East Islip, NY USA

Metastasis to the brain is a clinical problem in patients with breast cancer ${ }^{1-3}$. Between the brain and the breast resides the secondary lymphoid organ, the lymph nodes. We mined published microarray data ${ }^{4,5}$ to compare primary and metastatic tumor transcriptomes for the discovery of genes associated with metastasis to the lymph nodes in human breast cancer. We found that the zinc finger protein 367, encoded by ZNF367, was among the genes whose expression was most different in the lymph nodes metastases of patients with metastatic breast cancer as compared to primary tumors of the breast ${ }^{4}$. ZNF367 was also differentially expressed in the tumor cells of patients with triple negative breast cancer ${ }^{5}$. ZNF367 mRNA was present at increased quantities in lymph node metastatic tissues as compared to primary tumors of the breast. Importantly, expression of ZNF367 in primary tumors was significantly correlated with patient distant metastasis-free survival, in lymph node negative but not in lymph node positive breast cancer. Modulation of ZNF367 expression may be relevant to the biology by which tumor cells metastasize from the breast to the brain in humans with metastatic breast cancer.

Keywords: breast cancer, metastasis, brain metastasis, central nervous system metastasis, lymph node metastasis, the zinc finger protein $367, \mathrm{ZNF} 367$, systems biology of breast cancer, targeted therapeutics in breast cancer. 
One report described a 34\% incidence of central nervous system metastases in patients treated with trastuzumab for breast cancer ${ }^{2}$. More recently, the NEfERT-T clinical trial ${ }^{6}$ which compared administration of either neratinib or trastuzumab in conjunction with paclitaxel demonstrated that in a randomized, controlled setting, in breast cancer patients treated with neratinib, not only was the incidence of central nervous system distant metastasis significantly lower, the time to central nervous system metastasis was significantly delayed as compared to patients administered trastuzumab ${ }^{6}$. The alarmingly high rate of central nervous system metastasis described, as well as data, both anecdotal ${ }^{2}$ and from a randomized, controlled setting ${ }^{6}$ illustrating that treatment with trastuzumab may be associated with these events demands an enhanced understanding of the transcriptional makeup of brain metastatic tissues to support identification of therapeutic targets, whether they are treatment related or not. We performed a global comparative analysis of primary and metastatic tumors in human breast cancer ${ }^{4,5}$. We discovered differential and increased expression of the gene encoding the zinc finger protein 367, ZNF367, in lymph node metastatic tissues of patients with metastatic breast cancer.

\section{$\underline{\text { Methods }}$}

We used datasets GSE10893 ${ }^{4}$ and GSE38959 5 for this global differential gene expression analysis of brain metastatic breast cancer in conjunction with GEO2R. GSE10893 was generated using Agilent-011521 Human 1A Microarray G4110A technology with $n=71$ primary breast tumors and $n=7$ lymph node metastases from patients with breast cancer; analysis was performed using platform GPL887. GSE38959 was generated using Agilent-014850 Whole Human Genome Microarray 4x44K G4112F with $n=13$ samples of normal mammary gland ductal cells and $n=30$ samples of primary tumor cells from patients with triple negative breast cancer; analysis was performed using platform GPL4133. The Benjamini and Hochberg method of $p$-value adjustment was used for ranking of differential expression but raw $p$-values were used to assess statistical significance of global differential expression. Log-transformation of data was auto-detected, and the NCBI generated category of platform annotation was used. A statistical test was performed to evaluate whether ZNF367 gene expression was significantly different between primary tumors of the breast and lymph node metastases in humans with breast cancer using a two-tailed t-test. For Kaplan-Meier survival analysis, we used the Kaplan-Meier plotter online tool $^{7}$ for correlation of ZNF367 mRNA expression levels with distant metastasis-free survival in $n=4929$ breast cancer patients.

\section{$\underline{\text { Results }}$}

We performed global comparative transcriptome analysis of metastatic tumor tissues of patients with metastatic breast cancer using published microarray data ${ }^{4,5}$ to describe the transcriptional landscape of lymph node metastasis in human breast cancer in an unbiased fashion and for the discovery of novel therapeutic targets.

\section{ZNF367 is differentially expressed in lymph node metastases of patients with metastatic breast cancer.}

Through blind, systems-level analysis of published microarray data ${ }^{4}$, we identified the zinc finger protein 367, encoded by ZNF367, as a differentially expressed gene in the lymph node metastatic tissues of humans with breast cancer (Table 1). When sorting each of the genes expressed in lymph node metastases based on significance of difference as compared to primary tumors of the breast in patients with breast cancer, ZNF367 ranked 120 out of 18034 total transcripts (Chart 1), equating to 99.3\% differential expression. Differential expression of ZNF367 in the lymph node metastases of patients with metastatic breast cancer was statistically significant (Chart $1 ; p=1.11 \mathrm{e}-03$ ). 
To attempt to validate differential transcriptome-wide differential expression of ZNF367 in human breast cancer, we queried a second microarray datase $t^{5}$, here comparing primary tumor cells from patients with triple negative breast cancer to normal mammary gland ductal cells. Again, we identified ZNF367 as a differentially expressed gene in the tumor tissues of patients with breast cancer (Chart 2). When sorting each of the genes expressed in triple negative breast cancer tumor cells based on significance of difference as compared to normal mammary gland ductal cells, ZNF367 ranked 207 out of 45015 total transcripts (Chart 2), equating to $99.5 \%$ differential expression. Differential expression of ZNF367 in the primary tumor cells of patients with metastatic breast cancer was statistically significant (Chart 2; $p=2.74 \mathrm{e}-11$ ). Thus, differential expression of ZNF367, transcriptome-wide, in the tumor tissues of women with breast cancer was conserved across two independent microarray datasets, both in primary and metastatic tumor tissues.

\section{ZNF367 is expressed at lower levels in the lymph node metastases of patients with metastatic breast cancer.}

We obtained exact mRNA expression levels for ZNF367, in primary tumors of the breast and in lymph node metastasis of patients with lymph node metastatic breast cancer to determine direction and statistical significance of change in ZNF367 expression in lymph node metastatic tissues. We observed significantly lower expression of ZNF367 in the lymph node metastases of patients with breast cancer as compared to primary tumors of the breast: ZNF367 was expressed at $0.004 \pm 0.22$ arbitrary units (AU) in primary tumors of the breast, while it was expressed at $0.32 \pm 0.28$ AU in lymph node metastatic tissues. The difference in ZNF367 mRNA levels between primary tumors of the breast and lymph node metastatic tissues was statistically significant (Figure $1 ; p=0.000669$ ).

\section{ZNF367 expression is significantly correlated with survival outcomes in human breast cancer.}

We performed Kaplan-Meier survival analysis ${ }^{8}$ in 240 patients with lymph node negative breast cancer and 261 patients with lymph node positive breast cancer, to evaluate whether ZNF367 tumor expression was correlated with survival outcomes in human breast cancer. We observed a statistically significant correlation between primary tumor expression of ZNF367 and distant metastasis-free survival (DMFS) in patients with lymph node negative breast cancer (Figure 2). Lymph node negative patients whose primary tumors expressed low levels of ZNF367 possessed superior median DMFS as compared to lymph node negative patients whose tumors expressed high levels of ZNF367 (Figure 2). This difference in DMFS based on ZNF367 tumor expression in patients with lymph node negative breast cancer was statistically significant (Figure 2, Chart 3; logrank $p$-value: 0.012; hazard ratio: 2.54 (1.2-5.38)). DMFS was not correlated with primary tumor expression of ZNF367 in patients with lymph node positive breast cancer (Figure 2, Chart 3; logrank $p$-value: 0.51; hazard ratio: 1.17 (0.73-1.87)).

Thus, by mining published microarray data ${ }^{4,5}$ in an unbiased and systematic fashion, we identified the zinc finger protein 367, encoded by ZNF367, as among the genes whose expression was most different, transcriptome-wide, in the lymph node metastases of patients with breast cancer, when compared to primary tumors of the breast and in the primary tumor cells of patients with triple negative breast cancer when compared to normal mammary gland ductal cells; we observed significantly increased expression of ZNF367 in lymph node metastases as compared to primary tumors of the breast. Further, we found a significant correlation between ZNF367 expression and patient survival outcomes, as distant metastasis-free survival was significantly greater in patients whose primary tumors expressed low levels of ZNF367 as compared to patients whose primary tumors expressed high levels of ZNF367, in lymph node negative patients but not in lymph node positive patients. 


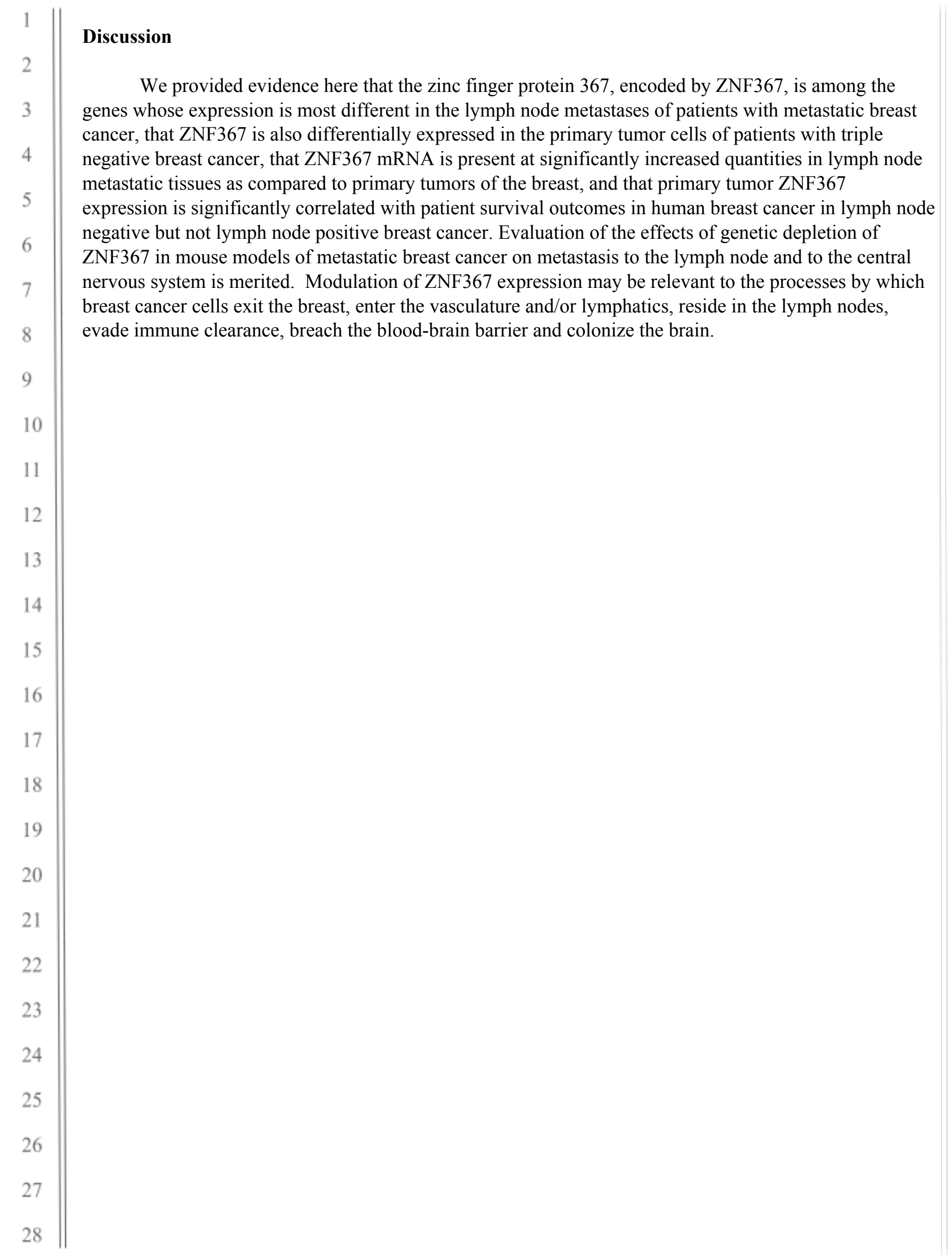




\section{References}

1. Lin, N.U., Amiri-Kordestani, L., Palmieri, D., Liewehr, D.J. and Steeg, P.S., 2013. CNS metastases in breast cancer: old challenge, new frontiers.

2. Bendell, J.C., Domchek, S.M., Burstein, H.J., Harris, L., Younger, J., Kuter, I., Bunnell, C., Rue, M., Gelman, R. and Winer, E., 2003. Central nervous system metastases in women who receive trastuzumab-based therapy for metastatic breast carcinoma. Cancer, 97(12), pp.2972-2977.

3. Tsukada, Y., Fouad, A., Pickren, J.W. and Lane, W.W., 1983. Central nervous system metastasis from breast carcinoma autopsy study. Cancer, 52(12), pp.2349-2354.

4. Weigman, V.J., Chao, H.H., Shabalin, A.A., He, X., Parker, J.S., Nordgard, S.H., Grushko, T., Huo, D., Nwachukwu, C., Nobel, A. and Kristensen, V.N., 2012. Basal-like Breast cancer DNA copy number cancer research and treatment, 133(3), pp.865-880.

5. Komatsu, M., Yoshimaru, T., Matsuo, T., Kiyotani, K., Miyoshi, Y., Tanahashi, T., Rokutan, K., Yamaguchi, R., Saito, A., Imoto, S. and Miyano, S., 2013. Molecular features of triple negative breast cancer cells by genome-wide gene expression profiling analysis. International journal of oncology, 42(2), pp.478-506.

6. Awada, A., Colomer, R., Inoue, K., Bondarenko, I., Badwe, R.A., Demetriou, G., Lee, S.C., Mehta, A.O., Kim, S.B., Bachelot, T. and Goswami, C., 2016. Neratinib plus paclitaxel vs trastuzumab plus paclitaxel in previously untreated metastatic ERBB2-positive breast cancer: the NEfERT-T randomized clinical trial. JAMA oncology, 2(12), pp.1557-1564.

7. Györffy, B., Lanczky, A., Eklund, A.C., Denkert, C., Budczies, J., Li, Q. and Szallasi, Z., 2010. An online survival analysis tool to rapidly assess the effect of 22,277 genes on breast cancer prognosis using microarray data of 1,809 patients. Breast cancer research and treatment, 123(3), pp.725-731. 


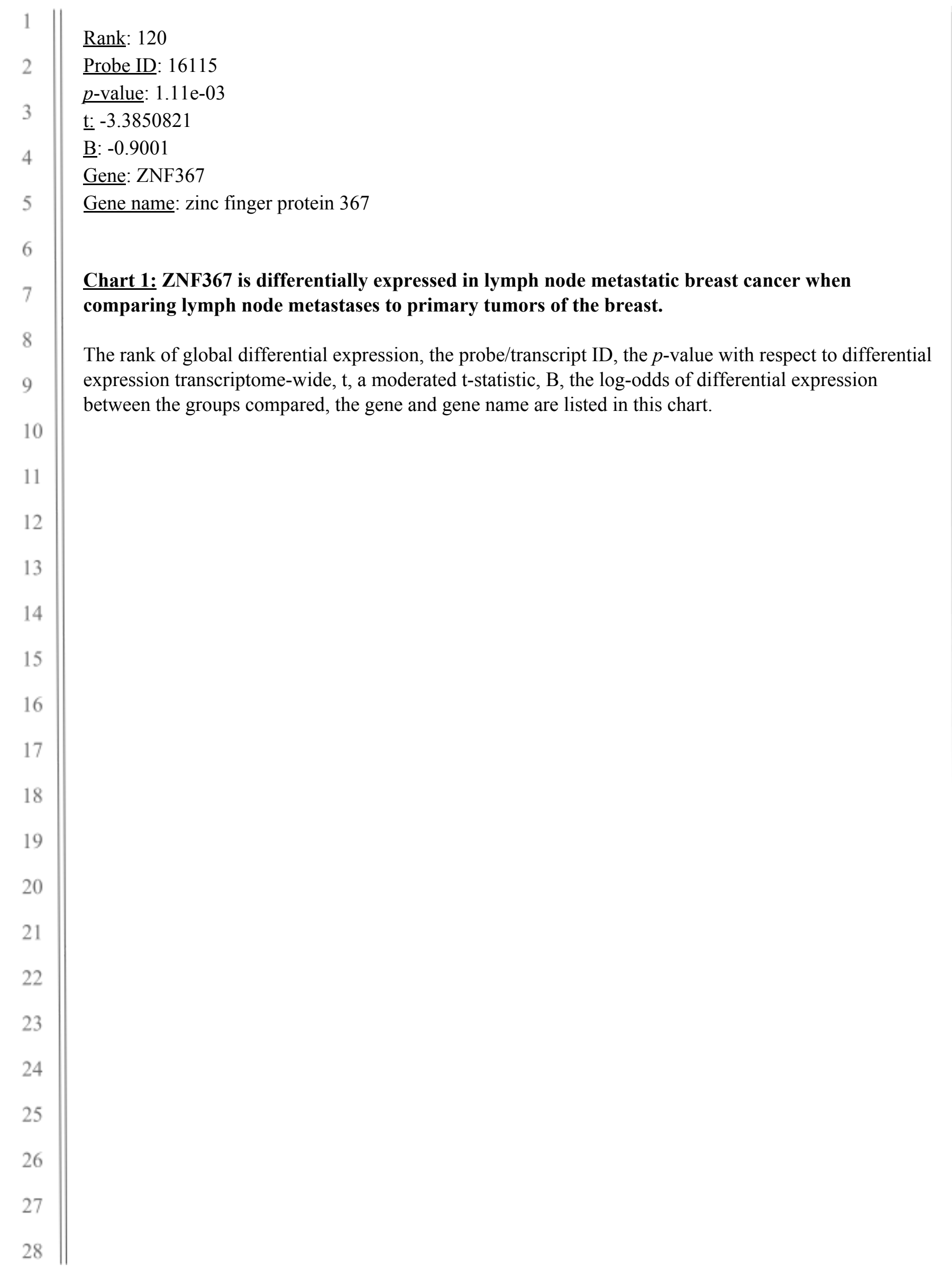




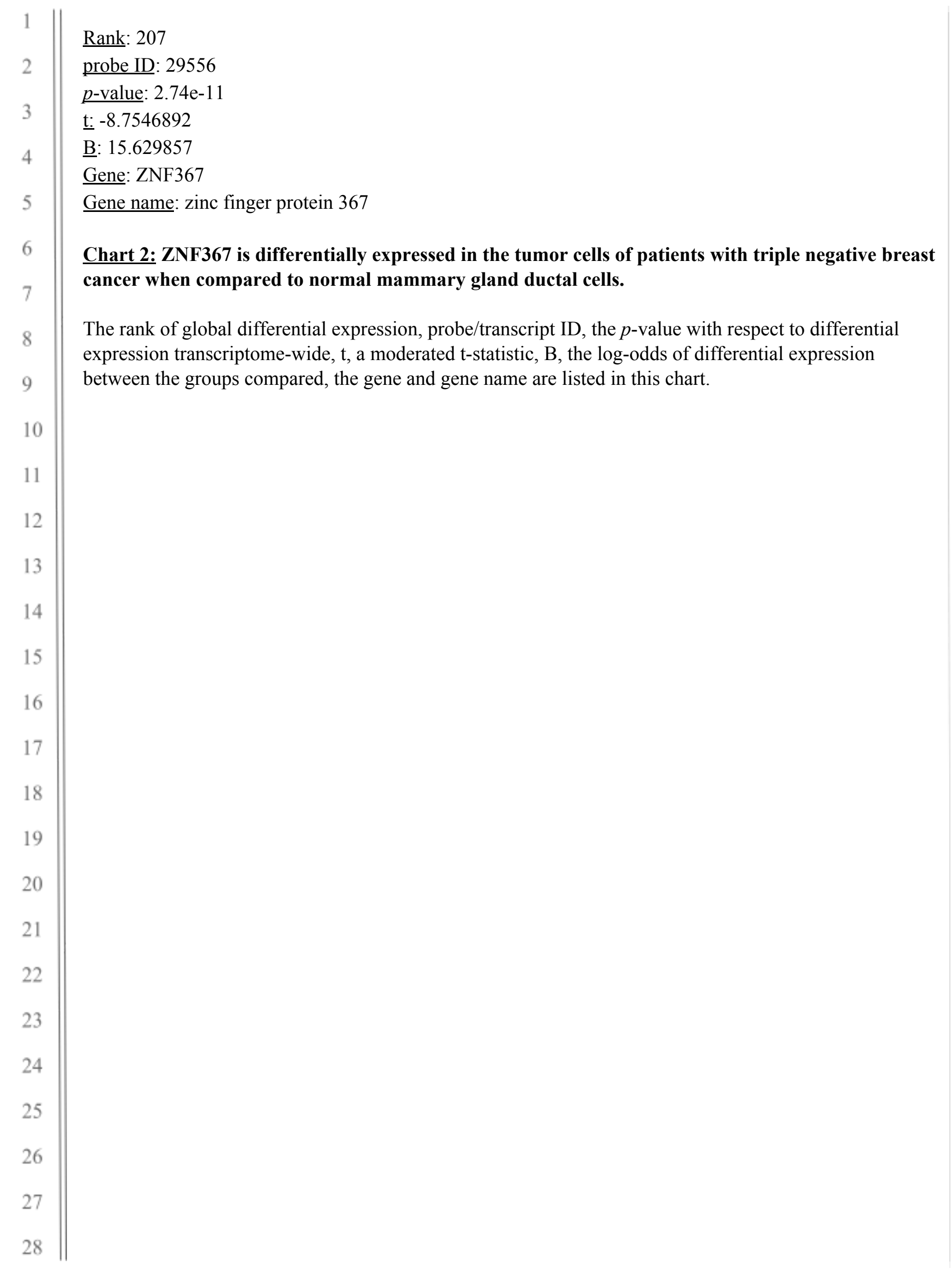




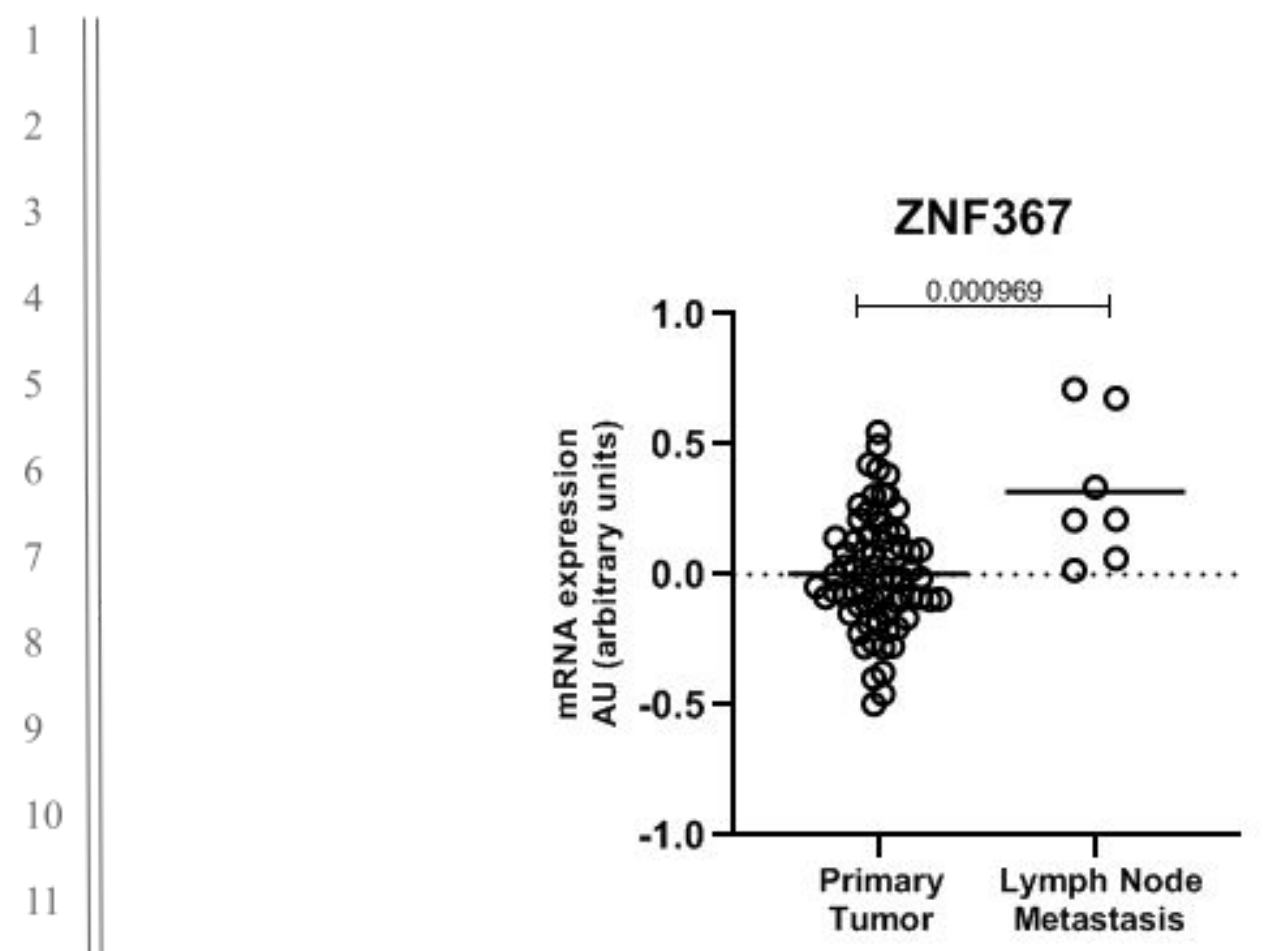

Figure 1: ZNF367 is expressed at significantly higher levels in the lymph node metastases of patients with metastatic breast cancer when compared to primary tumors of the breast.

The mRNA expression level of ZNF367 in primary tumors of the breast (left) and in lymph node metastases of women with metastatic breast cancer (right) is graphically depicted; the result of a statistical test evaluating significance of difference in ZNF367 expression between primary tumors and lymph node metastasis, a $p$-value, is $p=0.000969$. 


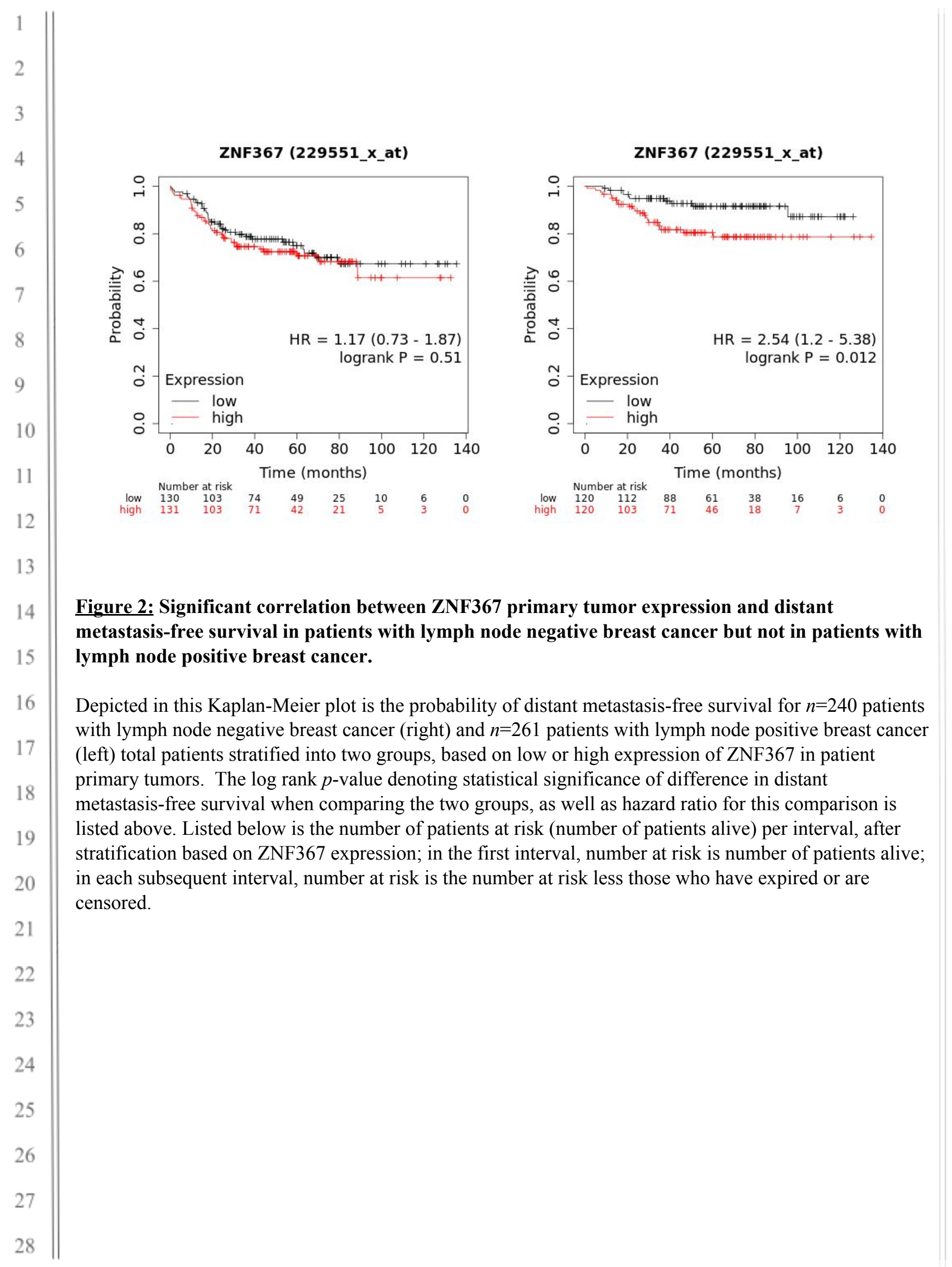

